\title{
Finding that osteoclasts repel osteoblast activity through Sema4D reveals novel target for bone-boosting therapies
}

$\mathrm{M}$ ost treatments for osteoporosis target osteoclastic bone resorption, but "bone-increasing drugs are wanted," states Hiroshi Takayanagi. In identifying semaphorin 4D (Sema4D) as a potent inhibitor of bone formation, in research now published in Nature Medicine, Takayanagi and colleagues hope that a new osteoanabolic treatment will ensue. Furthermore, the discovery of a new mechanism by which osteoclasts suppress osteoblast activity represents an advance in bone research.

"Bone resorption is linked to bone formation for maintenance of bone mass," says Takayanagi. This well-knownbut poorly understood-interrelation affects anti-resorptive and pro-formative therapeutic approaches alike, as perturbing one side of the bone remodeling equation can trigger 'corrective' action from the other side. Understanding how osteoclast activity is coupled to the anabolic effect of osteoblasts is thus key to improving outcomes in osteopenic disease.

"Classical coupling factors positively associate resorption with formation, and include IGF and TGF- $\beta$," continues Takayanagi. "Such factors regulate the transition from bone resorption into bone formation. However, we think that suppression of bone formation during bone resorption is also critical to maintain bone mass and quality." The team reached this conclusion after opting to study axon-guidance molecules (implicated in osteoclast-osteoblast communication). Expression analyses in mice revealed that among such molecules, "Sema4D is highly and specifically expressed by osteoclasts," as Takayanagi summarizes.

To probe the function of osteoclast Sema4D, the investigators analyzed the bone of Sema4D $D^{-/-}$mice. Bone volume, trabecular thickness, and bone strength were all greater in mice lacking Sema4D than in wild-type animals. Nevertheless, despite exclusive osteoclast expression of Sema4D, osteoclast parameters were not

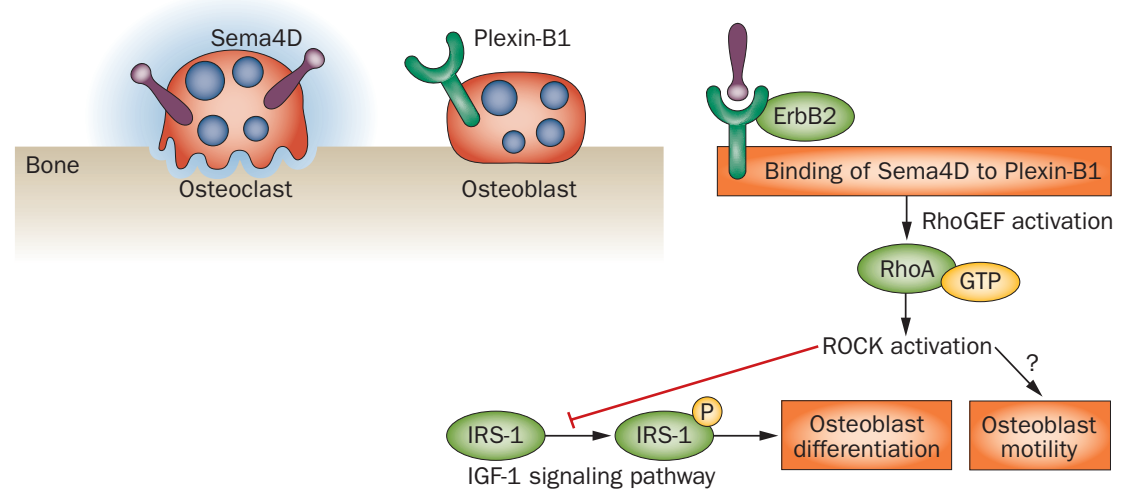

Osteoblast activity is suppressed in the vicinity of osteoclasts by Sema4D-Plexin-B1 interaction, which inhibits osetoblast differentiation via interference in the IGF-1 signaling pathway, and promotes osteoblast motility by an unclear mechanism.

different between wild-type and Sema4D ${ }^{-/-}$ mice. Instead, the osteoblast surface and rate of bone formation were increased in the knockout mice.

Osteoblasts differentiate from osteoprogenitor cells, whereas myeloid progenitors can become osteoclasts. Adoptive transfer of bone marrow (containing osteoclast precursors) from Sema $4 D^{-/-}$mice to wild-type animals resulted in high bone mass in the recipients; bone mass in Sema4D-deficient mice engrafted with wild-type bone marrow was normal. Furthermore, co-culture cell studies revealed that Sema $4 D^{-/-}$osteoclasts promote bone formation, and a Sema4D-specific antibody boosted bone formation in the presence of wild-type osteoclasts. These data suggest that osteoclasts produce factors that promote bone formation, but that the existence of these factors is imperceptible in the dominant-negative presence of Sema4D, which the osteoclasts also produce.

Osteoblasts, the researchers found, express the Sema4D receptor Plexin-B1, and $P l x n b 1^{-1-}$ mice have a similar bone phenotype to Sema4D ${ }^{-/-}$mice. Further studies indicated that after Sema4D binds to Plexin-B1, the kinase ErbB2 assists in the activation of RhoA and the downstream kinase ROCK, leading to suppression of IRS-1 phosphorylation (in the IGF-1 signaling pathway), and inhibition of osteoblast differentiation.
Sema4D-induced RhoA activation was also implicated in increased osteoblast motility in vitro, although the fine detail of this effect remains unclear. In vivo, a 'quiescent surface' can usually be found between osteoclast and osteoblast surfaces. Bone tissue from Sema4D ${ }^{-/-}$or Plxnb1 $1^{-/-}$ mice, however, had few such surfaces, and osteoblasts could be detected in close proximity to osteoclasts. Thus, the Sema4D-Plexin-B1 axis mediates spatial regulation of bone remodeling.

To test the effect of targeting Sema4D, Takayanagi and colleagues treated ovariectomized mice with an anti-Sema4D antibody, finding that it promoted bone formation without affecting resorption, and was effective as either prophylactic or therapeutic treatment. Now, their focus will turn to developing a suitable antibody for trial in humans, as well as to testing anti-Sema4D antibody in models of other diseases, such as arthritis.

Emma Leah

Original article Negishi-Koga, T. et al. Suppression of bone
formation by osteoclastic expression of semaphorin 4D.
Nat. Med. doi:10.1038/nm.2489
Further reading Lewiecki, E. M. New targets for intervention
in the treatment of postmenopausal osteoporosis Nat.
Rev. Rheumatol. 7, 631-638 (2011) | Edwards, J. R. \&
Mundy, G. R. Advances in osteoclast biology: old findings
and new insights from mouse models. Nat. Rev. Rheumatol.
$7,235-243$ (2011)

